\title{
Appropriation du français en contexte plurilingue africain : le nouchi dans la dynamique sociolinguistique de la Côte d'Ivoire
}

\author{
Jean-Baptiste Atsé N'Cho ${ }^{1,1}$ \\ ${ }^{1}$ Université de Bouaké, Département des Sciences du Langage et de la Communication, 01 BP V 18 \\ Bouaké 01, Côte d'Ivoire
}

\begin{abstract}
Résumé. La Côte d'Ivoire est l'un des pays d'Afrique linguistiquement hétérogène où aucune de ses langues locales n'a le statut de langue nationale ou ne sert de moyen de communication interethnique. L'objectif de cette contribution est de montrer comment, dans le contexte du plurilinguisme ivoirien, le nouchi qui, autrefois était utilisé comme un code secret par les jeunes des quartiers populaires d'Abidjan, s'étend désormais dans toutes les sphères de la vie privée et publique du pays. Après analyse, l'étude révèle que cette variété argotique joue un rôle véhiculaire indéniable au point de s'imposer comme la langue nationale de la Côte d'Ivoire. S'appuyant sur un corpus de mots et d'expressions nouchi extraits d'affiches publicitaires et de chansons zouglou, l'approche théorique de cette étude relève de la sociolinguistique variationniste et descriptiviste.
\end{abstract}

\begin{abstract}
Appropriation of french in an african plurilingual Context: the nouchi in the sociolinguistic dynamics of Côte d'Ivoire. Côte d'Ivoire is one of the linguistically heterogeneous countries in Africa where none of its local languages has the status of a national language or serves as a means of interethnic communication. The purpose of this contribution is to show how, in the context of Ivorian plurilingualism, the nouchi, which was formerly used as a secret code by young people in the neighborhoods of Abidjan, now extends in all spheres of private and public life of the country. After analysis, the study reveals that this slang variety plays an undeniable vehicular role to the point of imposing itself as the national language of Côte d'Ivoire. Rely on a body of nouchi words and expressions taken from
\end{abstract}

${ }^{1}$ Corresponding author : jbatse@yahoo.fr 
advertising posters and zouglou songs, the theoretical approach of this study is the Sociolinguistics variationist and descriptive.

\section{Introduction}

L'appropriation du français en contexte plurilingue africain, comme c'est le cas de la Côte d'Ivoire, a été l'objet de nombreuses recherches et publications et constitue toujours une source inépuisable pour les recherches en Sciences du langage. La coexistence du français avec la mosaïque des langues locales ivoiriennes, où aucune d'entre elles ne joue le rôle de langue nationale, a favorisé la véhicularité de différentes variétés de français dont le nouchi comme moyen de communication interethnique.

Autrefois considéré comme la langue des voyous et des jeunes délinquants des quartiers populaires d'Abidjan, le nouchi occupe désormais toutes les sphères de la vie publique et privée en Côte d'Ivoire. Même si de nos jours, le nouchi n'a pas encore totalement acquis un statut de code national, comment arrive-t-il à s'imposer aux Ivoiriens au point de devenir la langue nationale?

L'objectif de cette contribution est de montrer comment le nouchi est devenu populaire et est parvenu à s'imposer dans une Côte d'Ivoire (ethno)linguistiquement hétérogène. Conformément à cette objectif, nous nous sommes inspiré de la sociolinguistique variationniste et descriptiviste comme approche théorique telle que développée par Walter (1988), Dumont et Maurer (1995) et Gadet (2003) dans leurs différents écrits. Quant à méthodologie de collecte de données, elle s'appuie sur un corpus de mots et d'expressions nouchi recueillis in situ et par observation auprès de locuteurs parlant cette variété du français. La majeure partie de ces mots et expressions est extraite d'affiches publicitaires et de chansons zouglou [1]. Dans un premier temps, l'article présentera la situation sociolinguistique de la Côte d'Ivoire. Tout le reste du travail mettra l'accent sur le dynamisme du nouchi et étudiera son cas sur son avenir en tant que future langue nationale du pays.

\section{La situation sociolinguistique et la réalité du plurilinguisme en Côte d'Ivoire}

\subsection{Les grandes familles linguistiques et les langues ivoiriennes transfrontalières}

Les recherches linguistiques dénombrent soixante langues ivoiriennes dont certaines se retrouvent hors des frontières du pays.

\subsubsection{Les grandes familles linguistiques de la Côte d'Ivoire}

Selon la classification de Greenberg (1963) [2] et en nous fondant sur les clivages Nord/Sud/Est/Ouest, les grandes familles linguistiques en Côte d'Ivoire se répartissent entre quatre (4) groupes :

- Le groupe GUR ou VOLTAÏQUE (senoufo, koulango, lobi, gurunsi, etc.) avec 14 langues ;

- Le groupe KROU (bété, dida, neyo, wobè, etc.) avec

16 langues

- Le groupe KWA ou AKAN (baoulé, agni, akyé, abidji,n'zima, etc.) avec 17

langues ; 
- Le groupe MANDÉ (malinké, yacouba, dioula, gouro, etc.) scindé en deux groupes avec 13 langues. Quelques-unes des langues sont représentées sur la carte suivante avec leurs zones d'extension.

LES GROUPES ETHNO - CULTURELS DE CÔTE D'IVOIRE

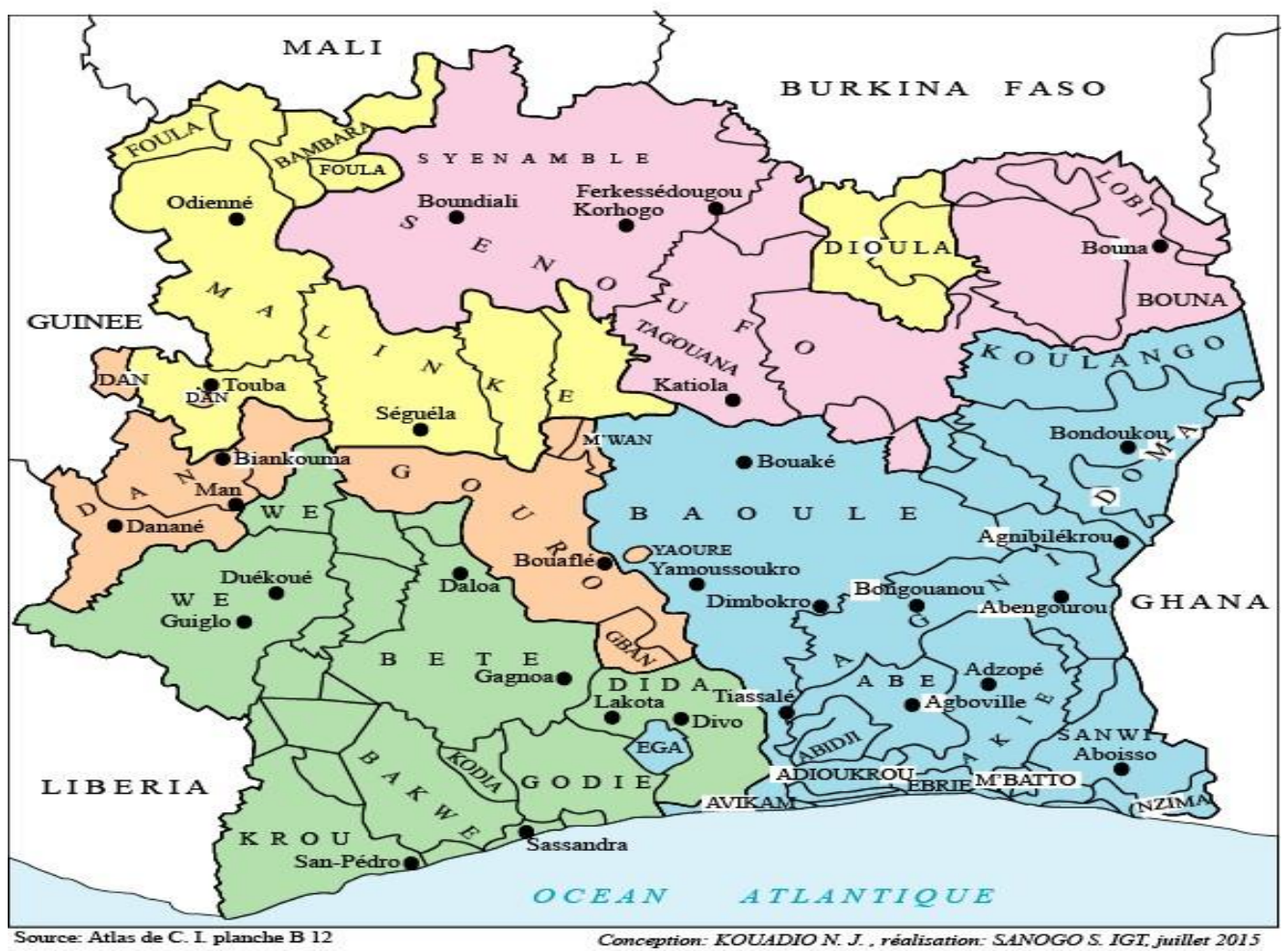

\section{$\square$ Groupe Mandé du Nord \\ $\square$ Groupe Mandé du Sud \\ Groupe Krou \\ Groupe Kwa (Akan) \\ Groupe Voltaĩque (Gur)}

Carte 1: Les quatre groupes ethnolinguistiques de Côte d'Ivoire

Source : Atlas de Côte d'Ivoire, Conception Kouadio N'Guessan, J. Réalisation Sanogo,

S. - IGT LATIG (juin 2007)

\subsubsection{Les langues ivoiriennes transfrontalières ou le multilinguisme regional [3]}

Une langue transfrontalière est une même langue, parlée par un groupe ethnique, une tribu ou une nation, située dans une aire géographique répartie entre deux ou plusieurs pays reconnus internationalement et ayant une frontière terrestre ou maritime commune (Cf. Wikipédia). C'est ce que Chaudenson (2000) appelle multilinguisme régional, c'est-à-dire la présence des mêmes langues dans plusieurs pays. En Côte d'Ivoire, toutes les quatre familles linguistiques ont chacune des langues qui font partie $\mathrm{du}$ multilinguisme regional ; elles transcendent donc les frontières ivoiriennes. Nous en dénombrons 16 au total [4]. Parmi elles, le manding [5] sert de véhiculaire régional, 
car, en plus d'être parlé en Côte d'Ivoire, il est présent au Burkina Faso, au Ghana, en Guinée et au Mali. Le Ghana reste le pays voisin qui partage le plus de langues avec la Côte d'Ivoire avec 10 langues (abron, agni-baoulé, birifor, degha, koulango, ligbi, lohron, manding, n'zima et senoufo).

\subsubsection{Les langues véhiculaires}

Louis-Jean Calvet définit une langue véhiculaire comme étant "une langue utilisée pour la communication entre des groupes qui n'ont pas la même première langue » (Calvet, 1993, p. 40). Prenant l'exemple de Dakar, la capitale du Sénégal, il démontre que «des gens dont le wolofn'est pas la première langue l'utilisent pour communiquer avec d'autres personnes qui n'ont pas la même première langue qu'eux» (Calvet, 1993, idem).

En Côte d'Ivoire, en plus du français (la langue officielle), les linguistes ivoiriens ont choisi, pour un besoin d'équilibre en fonction des familles linguistiques, une langue servant comme véhiculaire dans chaque famille linguistique. Ce sont : l'agnibaoulé [6] pour le groupe KWA, le bété pour le groupe KROU, le mandingue (dioula) pour le groupe MANDE et enfin le senoufo pour le groupe GUR. Néanmoins, il est rare - excepté la langue dioula - de voir en Côte d'Ivoire des gens utiliser ces langues dites véhiculaires pour communiquer avec d'autres personnes qui n'ont pas la même première langue qu'eux. Seul le français joue le rôle de langue véhiculaire entre les Ivoiriens qui n'ont pas la même première langue. Selon Kube, «la véhicularité du français est assez importante en milieu urbain où la population multiethnique se sert de cette langue comme moyen de communication interethnique ». Quant aux langues dites véhiculaires ivoiriennes, l'auteur affirme que leur "véhicularité n'est pas importante en Côte d'Ivoire [car] leur nombre est très grand et aucune des langues n'a pu s'imposer en tant que véhiculaire (mis à part le dioula servant, sur beaucoup de marchés ivoiriens, de langue de commerce)» (Kube, 2005, p. 60).

\subsection{Le français et ses variétés en Côte d'Ivoire}

Le statut du français en tant que langue officielle en Côte d'Ivoire reste sérieusement secoué par sa coexistence avec les langues locales, donnant ainsi naissance à différentes variétés de français observées sur le territoire. Certains chercheurs comme Lafage (1991, 1996 ; 1998), Kouadio (1999 ; 2006) et Boutin (2002) parlent de variétés basilectale, mésolectale et acrolectale. Kouadio ${ }^{7}$ donne les raisons de cette catégorisation:

Au départ, sur la base d'un travail épistémologique hâtif, on avait postulé une répartition tripartite de ces pratiques en acrolecte, mésolecte et basilecte, corrélés chacun avec des strates sociales, elles, définies par leur rapport à l'éducation scolaire. Suivant ce postulat, les élites de la société étaient censées parler la variété acrolectale, les personnes moyennement scolarisées la variété mésolectale et les peu ou pas du tout scolarisés la variété basse ou le français populaire ivoirien (fpi), anciennement "petit-nègre", "français de Moussa" ou encore "français de Treichville" [7].

Pour nous, en parlant de variétés de français en Côte d'Ivoire, nous abondons dans le même sens que Kouamé, où l'on retrouve tout simplement «une forme standard qui se cantonne aux écoles et à la vie administrative et deux formes véhiculaires (le français ivoirien et le nouchi) qui explorent toutes les autres sphères de la vie privée et publique » (Kouamé, 2013, p. 70). Notre étude se fondant essentiellement sur cette dernière variété, nous allons chercher à comprendre tout ce qui concourt à sa naissance et à son dynamisme. 


\subsection{Le nouchi}

Apparu et pris réellement forme dans les années 1980, le nouchi est l'une des variétés de français en Côte d'Ivoire qui, à l'oreille, se rattache au français, mais à forte dominante africaine. L'on dit même souvent du nouchi qu'il serait une pure invention des jeunes voyous, délinquants ou déscolarisés des quartiers précaires d'Abidjan. En effet, plusieurs hypothèses concourent à l'étymologie du terme "nouchi". Pour Lafage Suzanne, ce terme dont la première trace se situe en 1977 tire son origine de deux mots dioula : «nún : nez et mii : poil, traduisant "poil du nez" » et aurait servi à l'époque comme synonyme de "voyou" (Lafage, 1991, p. 97). Quant à Kouadio N'guessan, ses informateurs lui auraient affirmé en 1991 que nouchi serait un mot soussou (une langue mandée parlée en Guinée) et aurait pris naissance à Adjamé, l'un des quartiers populaires d'Abidjan où vit une forte communauté soussou (Kouadio, 1991, p. 373). De ces deux sources, l'hypothèse de Lafage selon laquelle le terme nouchi aurait pour synonyme de "voyou" nous parait très probable, tout simplement parce qu'à cette époque-là, les Ivoiriens, très friands de films Westerns que diffusait leur seule et unique chaîne de télévision tous les dimanches à partir de $14 \mathrm{~h}$, identifiaient toujours les téméraires, les caïds et même les braves par leurs moustaches. Ainsi, tous ceux qui portent des moustaches dans les films western (ou far West) sont considérés comme des Noussis [8], c'est-à-dire des durs à cuire, des caïds, des bandits, et en même temps, des braves. C'est ce qui amène Suzanne Lafage (1991) à dire que le mot nouchi a été utilisé en ses débuts pour désigner des jeunes de la rue vivant du vol à la tire; lesquels se prennent pour des caïds. Une autre version qui nous a été racontée au cours de nos enquêtes nous fait croire que le mot "nouchi" serait la déformation de "nous ici-là" pour designer "chez nous", "notre territoire", "notre espace à nous". Ce serait donc par le chuintement de "ici" pour "chi" qu'on aurait abouti au mot nouchi qui se réfèrerait à "notre façon de parler à nous". Une chose est sûre, le nouchi, en plus d'être une pratique langagière (des jeunes, des voyous, etc.) est une marque identitaire. Les locuteurs du nouchi eux-mêmes s'identifient à un lieu, un quartier, un territoire et se les revendiquent dans leurs manières de parler.

Pour sa classification, la littérature scientifique le caractérise comme un argot ou une langue des jeunes (Kouadio, 1990 ; Lafage, 1996 ; 1998), ou souvent comme une langue hybride ou un mélange de langues (Lafage, 1998). D'autres encore le considèrent comme une langue métissée ou une langue mixte (Queffelec, 2007). La spécificité du nouchi, c'est la capacité de ses locuteurs à se créer leurs propres règles grammaticales et à mettre à profit tous les procédés de créations lexicale, morphologique, syntaxique à partir d'une langue quelconque pour réinventer une langue propre à eux; ce qui fait dire à Kouadio [9] que

le nouchi se différencie des autres variétés par son vocabulaire caractérisé par :

- des emprunts massifs aux langues ivoiriennes et non ivoiriennes

- la création de mots hybrides ;

- la resémantisation de certains termes du français qui deviennent polysémiques ;

- la création de nombreux synonymes ;

- l'usage de tous les procédés linguistiques de création lexicale ;

- l'utilisation consciente des figures de style telles que la métaphore, la métonymie, l'extension et le rétrécissement de sens, etc.

Cela se solde par un lexique foisonnant, instable parce que constamment renouvelé, mais dont certains mots, locutions et tournures sont si expressifs qu'ils finissent par paraître emblématiques de cette façon tout ivoirienne de jouer avec la langue française.

Alors comment un tel phénomène linguistique arrive-t-il à s'imposer à tous les Ivoiriens? 


\section{Les facteurs du dynamisme du nouchi}

Le nouchi n'est plus de nos jours cet argot crypté parlé principalement par les jeunes de la rue, les délinquants et autres loubards, mais un parler qui explore toutes les couches de la société ivoirienne et qui, depuis lors, a fait son entrée dans les lycées et collèges et à l'université. Selon Aboa (2011, p. 47), «son extension ne s'arrête pas aux jeunes. Elle touche aussi les parents quelle que soit leur condition sociale, qui l'utilisent dans la communication avec leurs enfants ». Si aujourd'hui le nouchi gagne du terrain en Côte d'Ivoire, cela est dû à plusieurs facteurs [sociolinguistiques]. Le premier facteur est l'absence d'une langue véhiculaire à l'échelle nationale. En réalité, la très grande complexité linguistique que nous rencontrons en Côte d'Ivoire et surtout l'utilisation qui en est faite prouvent qu'il y a une véritable absence de langue véhiculaire d'extension nationale. En dehors de l'association agni-baoulé et le manding sous la forme spécifique du dioula qui pourraient jouer ce rôle, force est de reconnaitre que toutes les autres langues ivoiriennes sont considérées comme des "langues de moindre diffusion" et se limitent à leurs zones naturelles d'extension (Sangaré, 2009, p. 221). Dans un tel environnement, les jeunes non scolarisés ou déscolarisés qui sont d'origine ethnique différente et qui parlent des langues premières différentes (Lafage, 1991, p. 97 ; Kube, 2005, p. 25) vont, pour répondre à leur besoin de communication dans une ville cosmopolite comme Abidjan, créer ce mode langagier (le nouchi) qui dépasse les clivages ethniques.

Le second facteur pourrait s'expliquer par le couple français / langues vernaculaires ivoirienne. L'État de Côte d'Ivoire, en choisissant le français comme langue officielle de par ses différentes constitutions, développe une politique linguistique exoglossique [10], mettant le français en coexistence avec les langues vernaculaires ivoiriennes. De cette coexistence où n'émerge aucune langue ivoirienne véritablement dominante, les études montrent qu'un Ivoirien, en plus de sa langue maternelle qu'il acquiert comme première langue, a le français comme langue seconde ou vice-versa. "Il se pose alors la question de la cohabitation de ces langues dans l'espace culturel ivoirien, celle de la gestion spontanée ou raisonnée que les Ivoiriens font de ce bilinguisme francoivoirien, au demeurant déséquilibré au profit du premier terme » (Kouadio, 2000, p. 199). Pour nous, un tel bilinguisme pourrait favoriser le développement linguistique et cognitif des sujets par la fabrication d'un code simplifié à l'apprentissage réussi de deux langues ou même plus. Le nouchi en serait un cas d'espèce.

Le troisième facteur du dynamisme du nouchi est l' "appropriation » du français par les Ivoiriens. En effet, le français est une langue étrangère importée et imposée aux Ivoiriens par l'administration coloniale comme une nouvelle langue, une nouvelle culture, une nouvelle identité. Dans les pays francophones d'Afrique, il jouit d'un prestige jamais démenti ni contesté. Se l'approprier serait une sorte d'ascension sociale ou d'ouverture au monde, mais surtout un moyen d'obtenir une place dans la société coloniale. En plus, lorsque le français est utilisé dans des situations non officielles, par exemple dans la communication quotidienne, il doit rendre des contenus d'un tout autre cadre culturel. C'est ce que Manessy appelle la sémantaxe ; phénomène qu'il caractérise de la manière suivante :

Les particularités du français africain ne se montreraient pas uniquement dans le lexique sous forme d'emprunts de langues africaines, mais aussi dans la sémantique. Dans la pratique linguistique des Africains se manifesterait une "manière africaine de voir les choses". Le français se développerait dans un environnement culturel différent et n'y serait pas utilisable de la même manière (Manessy, 1994, p. 86-87). 
Mais pour Wald,

L'appropriation du français en Afrique sera complète si une variété autonome se stabilise et si l'utilisation du français n'est plus mesurée à la norme extérieure. Celle-ci serait cependant encore très présente actuellement surtout à l'école comme une sorte de "vigilance métalinguistique" et comme moyen de catégorisation sociale des locuteurs (Wald, 1994, p. 122).

Tout ceci montre que l'appropriation du français en contexte plurilingue africain pourrait se déterminer par un " marquage identitaire » spécifique (Moreau, Thiam et Bauvois, 1998).

Enfin, un autre facteur qui a fortement participé au dynamisme du nouchi a été, sans aucun doute, sa vulgarisation par les mass-médias; toute la presse ivoirienne confondue aussi bien qu'avec le cinéma, les chansons zouglou et reggae. Le nouchi a véritablement connu une ascension fulgurante depuis l'ouverture du pays à la démocratie pluraliste en 1990. "En cette année-là, plus d'une quarantaine de titres de journaux se partagent le marché ivoirien de la presse écrite (quotidiens, hebdomadaires, magazines, etc.), mais aucune de ces publications n'est en langue nationale » (Kouadio, 2000, p. 201). Quelques directeurs de publications vont jusqu'à barrer la une de leurs journaux en utilisant le nouchi (Voir les Images 2 dans les pages suivantes). Même la littérature écrite s'y met. Tous les numéros de la collection Aya de Yopougon de Marguerite Abouet et Clément Oubrerie destinée à la littérature enfantine sont entièrement écrits en nouchi avec des personnages et des images bien illustrés. Quant aux artistes zouglou et de reggae, le nouchi devient leur principal support linguistique. Les titres C'est pas da ni bloh (pour « ce n'est ni de la vantardise, ni du bluff ») du reggaeman Serge Kassy et Premier Gaou (pour « Dupé pour la première fois ») du groupe zouglou Magic System sont des exemples parmi tant d'autres.

Au début, dans ces musiques, l'apport se faisait par l'intégration de quelques mots nouchi expliqués plus ou moins maladroitement. Puis, au milieu des années 88/90, une certaine mouvance Reggae (Wabi Spider, Serges Kassi et surtout Tangara Speed Godha) va proposer les premiers énoncés complets en nouchi [11].

En guise d'exemple, prenons l'extrait d'une chanson du groupe Les Potes de la rue intitulée Ziopin [12], une chanson qui a eu beaucoup de succès en 1996 et dans laquelle l'auteur dénonçait le tribalisme et l'intolérance :

- Qui fait ça ? Faut pas fasser hein, nous s'amuser! Refrain : - Tribalisme n'est pas bon o!

- Baoulé dit qu'Agni est soûlard, Agni dit que Baoulé est soûlard

Entre les deux-là qui $\mathrm{i}$ boit fort? Refrain

- Bêlard dit que ler sont digba, les Loubards disent i sont digba

Entre les deux-là qui a gnaga ? Refrain

- Abidji dit que ler sont sorcier, Adioukrou dit que ler sont sorcier

Entre les deux-là qui peut boi acide ? Refrain : - Sorcellerie n'est pas bon o !

- Dioula dit que Tagbana a petit pied, Tagbana dit que Dioula a petit pied

Entre les deux là pour qui est tchrin ? Refrain

- Gagou dit que Dida a digba mollet, Dida dit que Gagou a digba mollet

Entre les deux-là pour qui est agbôlô ? Refrain

- Pygmée dit que Niaboua est court, les Niaboua dit que Pygmée est court

Entre les deux-là qui est plus grand ? Refrain

- Mauritanien dit qu'il est blanc, Albinos dit qu'il est blanc aussi

Entre les deux-là qui est blanc teint clair? Refrain

Essai de traduction: 
- Attention! Ne nous prenez pas au sérieux, c'est de la plaisanterie !

- Le tribalisme est dangereux!

- Les Baoulé traitent les Agni d'ivrognes, les Agni en font autant

Où est donc la vérité ?

- Les porte-faix prétendent qu'ils sont musclés et forts, les Loubards disent qu'ils sont les plus musclés et les plus forts

Où est donc la vérité ?

- De ces deux ethnies (Abidji et Adioukrou), laquelle est la plus forte en sorcellerie ?

- Les Dioula disent que les Tagbana ont de petits pieds, les Tagbana pensent plutôt que ce sont les Dioula qui ont de petits pieds

Où est la vérité ?

- Les Gagou prétendent que les Dida ont des mollets difformes, les Dida pensent le contraire

Où est donc la vérité ?

- Les Pygmées disent que les Niaboua sont de petite taille, les Niaboua prétendent le contraire

Où est la vérité ?

- Les Mauritaniens disent qu'ils sont des Blancs, les Albinos disent qu'eux aussi sont des Blancs

Lequel est le plus Blanc des deux?

À la lecture de ce texte, les quelques caractéristiques du français populaire ivoirien (FPI) et du nouchi apparaissent :

a) les caractéristiques relevant du FPI :

- l'absence d'article devant les noms d'ethnies : Baoulé, Agni, Gagou, Dida, Tagbana,

Dioula, Adioukrou, Abidji, etc.;

- des prononciations approximatives : « fasser » (fầcher), «ler » (leur);

- l'emploi des unités dicto-modales d'origine africaine telles que « de ! o !»;

- l'emploi particulier de "même » qui marque ici le superlatif;

- l'emploi du présentatif «-là » fonctionnant comme élément à valeur anaphorique et emphatique ;

- la construction possessive avec «pour » dans «pour qui est agbôlô ? »;

- le pronom relatif qui en deux morceaux (qui + il) avec une prononciation approximative de il : « qui i boi

» $\mathrm{t}:$ «qui boit ? »

b) les caractéristiques relevant du nouchi :

II s'agit surtout ici de mots de vocabulaire : «bêlard » (porte-faix), « digba » (gros et musclé), « gnaga » (ici traduit par la « force »), « tchrin » (mince), « agbôlô » (gros et difforme).

En plus de ces caractéristiques d'ordre linguistique, il faut ajouter que ces chansons sont chantées sur un rythme, un air, avec une mélodie typiquement ivoiriens, ce qui a pour effet de décomplexer et de sécuriser les chanteurs qui échappent ainsi au style « chocobit [13]» décrié par l'ensemble des Ivoiriens. L'utilisation de cette sorte d'interlecte donne l'impression aux chanteurs qu'ils chantent en français sans y perdre, ni leur identité, ni leur culture. De son côté, le public, qu'il soit lettré ou analphabète non locuteur du français, s'y retrouve de quelque manière : le public lettré parce qu'il pratique toutes les variétés de français présentes en Côte d'Ivoire, le public analphabète, parce qu'il y retrouve des rythmes, des vocalisations propres au terroir (Kouadio, 2000, p. 202-203).

Au regard de tout ce qui précède, nous pouvons dire que le dynamisme même du nouchi, c'est sa particularité de s'inspirer de la vie politico-socio-culturelle ivoirienne et d'évoluer avec elle. Son vocabulaire, nourri de l'actualité, tire ses ressources dans les conversations quotidiennes des personnes de différentes classes sociales et/ou de différents groupes linguistiques. Sa vulgarisation est désormais plus étendue dans toute 
la société, notamment dans les domaines politique, médiatique, culturel et publicitaire (Voir chapitre 4.3).

\section{Les fonctions communicatives et identitaires du nouchi}

L'analyse des fonctions communicatives et identitaires du nouchi fait apparaitre un tableau très complexe. Sabine Kube (2005, p. 91 ; 133 et suiv.), dans son enquête sur le nouchi à travers une analyse d'attitudes linguistiques menée auprès des élèves en milieu scolaire, présente les fonctions actuellement remplies par ce parler argotique qui est le moyen de communication préféré des jeunes. Nous avons également mené, pendant près de trois ans, cette même enquête auprès de nos étudiants de Licence 2 du département des Sciences du Langage et de la Communication de l'Université de Bouaké au titre des années académiques 2014-2015;2015-2016 et 2016-2017, et ce, dans le cadre de l'unité d'enseignement intitulé : Géographie linguistique de la Côte d'Ivoire. Les propos de ces étudiants qui vont dans le sens de ceux des élèves enquêtés par Kube sont enrichissants de fort belles manières. Nous en reprenons quelques-uns.

\section{1 Le nouchi et les jeunes}

Dès son avènement, le nouchi servait de code secret aux jeunes de la rue et ceux qui le parlaient étaient catégorisés de voyous. Soro Solo en donne les raisons :

Le secteur de l'informel se divise en deux blocs : celui des patrons et celui des apprentis. Il fourmille de jeunes non-scolarisés et de déchets scolaires. La loi du milieu est implacable. Comme les mousses, les apprentis chauffeurs ou des petits métiers sont corvéables à souhait. Ils doivent soumission absolue et reconnaissance au patron qui leur offre "gracieusement" le savoir. Dans un tel univers où délation, chantage, punition corporelle et raison du plus fort constituent les règles du jeu, on a intérêt entre gens de condition égale à élaborer un code ésotérique pour échanger ses fragments d'info sans que les aînés n'en comprennent rien.

Ainsi, le nouchi bourgeonna et se développa dans les gares routières, les garages de mécanique auto, les ateliers de menuiserie, les hangars de marché, avant de gagner la rue tout entière puis les lycées et collèges.

$\mathrm{Vu}$ ses origines underground, le nouchi est forcément perçu par la bonne morale comme une langue de voyous (Soro Solo, 2003).

Le nouchi a renforcé ses positions dans le milieu des jeunes à tel point qu'il est devenu la première langue (Kouadio, 2006 : 177). Kube révèle ceci dans son enquête :

Le nouchi est, par excellence, l'alternative au français selon les élèves. [...] Nos résultats montrent que le nouchi remplit pour les élèves des fonctions communicatives et identitaires que ni le français qu'ils apprennent tous à l'école, ni les langues ivoiriennes de leur répertoire linguistique ne peuvent satisfaire et qui vont bien au-delà des fonctions attribuées à une langue des jeunes (Kube, 2005, p. 133).

$\mathrm{Vu}$ sous ces différents aspects, le nouchi peut être considéré comme une variété privilégiée des jeunes, d'une génération et d'une communauté linguistique qui le revendiquent au quotidien comme code de ralliement et comme moyen d'affirmation de leur esprit créateur et de leur volonté de liberté dans la société.

\section{2 Le nouchi et la communication interethnique}

Dans une Côte d'Ivoire linguistiquement hétérogène où aucune langue locale ne joue le rôle de langue véhiculaire à l'échelle nationale, la recherche scientifique désigne 
depuis longtemps le français comme la langue qui remplit cette fonction linguistique. C'est le cas de Kouadio quand il affirme que l'extraordinaire expansion du français, toutes variétés confondues, [qui] est devenu le premier véhiculaire du pays, à telle enseigne qu'aujourd'hui aucun secteur d'activités, aucun endroit, si reculé soit-il, n'échappent à la présence et à l'emploi du français (Kouadio, 2000 : 199). Parlant de variétés de français, la plus usitée est le nouchi qui, dans l'enquête de Kube, « est, aux yeux des élèves, un élément indispensable de l'éventail linguistique ivoirien. Il y remplit des fonctions communicatives dans lesquelles aucune autre variété de langue ne peut le remplacer» (Kube, 2005, p. 139). En guise d'exemple, l'auteur donne quelques témoignages d'élèves de lycées et collèges d'Abidjan et sa banlieue : «Le nouchi est né parce que tous les habitants ne pouvaient pas parler le français soutenu et parce qu'il n'y a pas de langue commune (Troisième, f, 15 ans)»; " Le nouchi est né pour que les conversations se font entendre de tout le monde et dans la même langue, en fait une seconde langue après le français (Troisième, f, 13 ans) »; "Nous nous comprenons plus [avec le nouchi], parce que les langues maternelles ne sont pas les mêmes (Troisième, f, 15 ans) »; "Le nouchi est né pour résoudre le problème de communication entre les différentes couches (Terminale, f, 19 ans) » (Kube, 2005, p. 140-141).

\section{3 Le nouchi, les médias, la politique, la culture et la publicité}

En plus d'être un moyen de communication interethnique, le nouchi est également et sans ambages la langue des médias, du politique de la culture et de la publicité. Pour s'en convaincre, il suffit d'étudier ce mélange respectif du français avec des langues nationales à travers quelques produits culturels que sont les affiches et campagnes publicitaires, les unes de journaux, les émissions de radio et de télévision, les livres, les chansons et les réseaux sociaux.

Ainsi, au niveau des médias, précisément dans les chaines de radios et de télévisions, le nouchi est bien audible. La Côte d'Ivoire compte deux chaînes de radios nationales (Radio nationale et Fréquence 2) et une centaine de chaînes privées réparties dans toutes les villes et communes. Elle compte également trois chaînes de télévision nationales (RTI 1, RTI 2 et RTI Bouaké) qui, en dehors de RTI Bouaké, émettent sur toute l'étendue du territoire et sur le bouquet Canal+ avec certaines chaînes privées depuis la capitale économique Abidjan. Presque tout l'espace radiophonique et télévisuel en Côte d'Ivoire diffuse des émissions favorisant et promouvant le nouchi. Les exemples sont multiples : les animateurs d'émissions comme «After work» sur Radio Nostalgie, « Djassa FM » animé par Julien Goualo (artiste musicien féru du nouchi) sur Fréquence 2, «C'midi » et « On s'éclate » sur la chaîne de télévision RTI 1, et d'autres émissions sur les radios et télévisions privées et communautaires (aussi appelées radios de proximité), etc. utilisent le nouchi à profusion.

$\mathrm{Au}$ niveau publicitaire, les compagnies de téléphones mobiles et les brasseries ivoiriennes ne sont pas en reste. Leurs messages en nouchi sont conçus généralement pour attirer à la consommation et l'adhésion des jeunes qui constituent un public potentiel. Pour les téléphones mobiles, par exemple, l'opérateur Orange-Côte d'Ivoire l'utilise dans son slogan «SOS crédit, quand ya drap \#170\# te soutra " (voir l'affiche à gauche des images 1 ci-dessous) pour dire «quand tu n'as plus de crédit sur ton compte \#170\# te dépanne ». Quant aux brasseries ivoiriennes, l'affiche à droite des images 1 ci-dessous le démontre : "Authentique comme les vieux pères d'ici. Connaisseur connait! ». En nouchi, le terme vieux père signifie «Aîné ou devancier [dans un corps de métier] » et l'expression proverbiale Connaisseur connait! qui pourrait se traduit par «c'est l'averti qui (re)connait les bonnes choses [ici par le goût] $»$. 

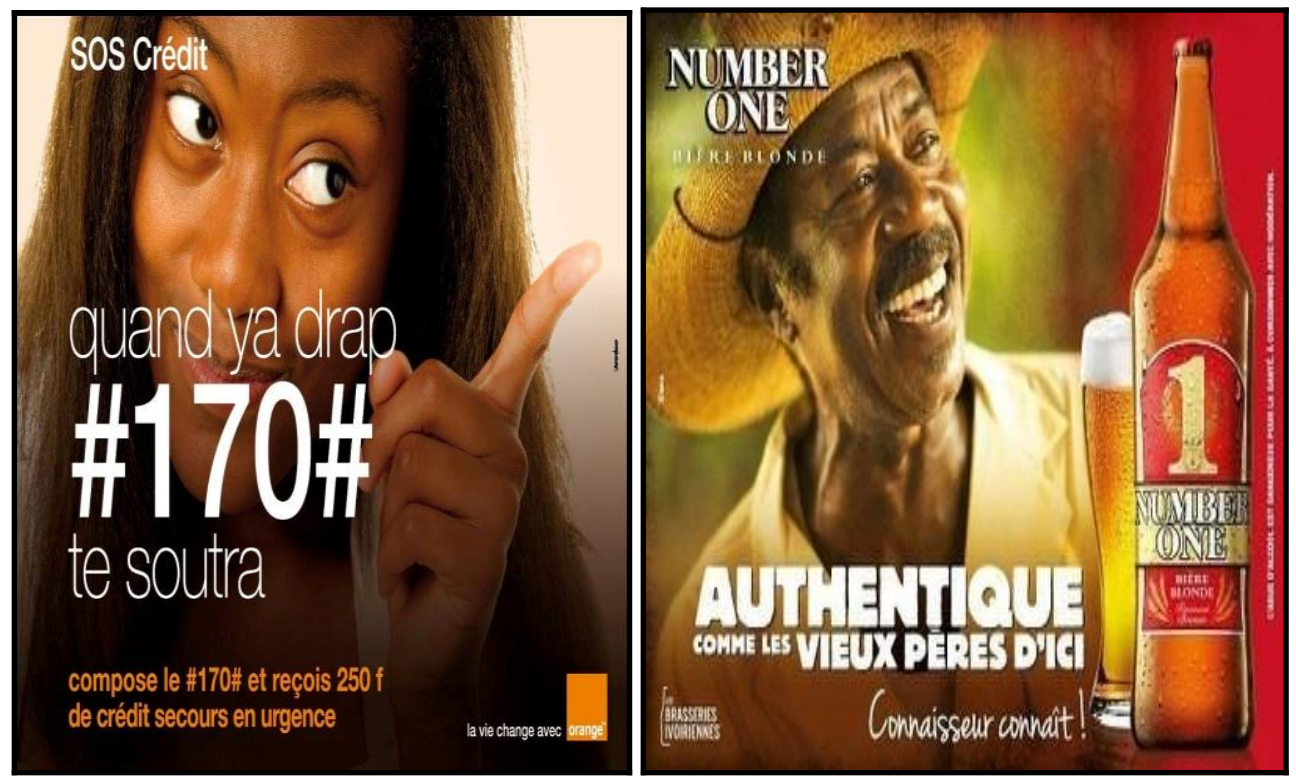

Images 1. Exemple d'affiches publicitaires

En ce qui concerne la presse écrite avec les journaux d'informations générales et la communication politique, le nouchi occupe une place importante. Ces deux exemples tirés des Unes de journaux le démontrent à travers les images 2. Pour le quotidien Nord-Sud, sa publication $\mathrm{N}^{\circ} 1286$ du 31 août 2009 retranscrit un discours du Président du Parti Démocratique de Côte d'Ivoire (PDCI), s'adressant à ses militants en nouchi en ces termes : "Plus de flôkô, les Refondateurs vont fraya », propos qui pourrait être traduits par «Il n'y aura plus de mensonges [dans la gestion de l'État], le régime des Refondateurs [le Front Populaire Ivoirien de Laurent Gbagbo au pouvoir de 2000 à 2011] va fuir (débarrasser le plancher) ». Quant au quotidien L'Intelligent d'Abidjan dans sa parution $\mathrm{N}^{\circ} 2382 \mathrm{du} 4$ novembre 2011, parlant des élections législatives de 2011, barre sa Une par l'information suivante : NON à des élections koutcha et wouya-wouya. "Koutcha » et «wouya-wouya » sont deux termes malinké qui se traduisent respectivement par «truquées" et «chose sans importance » comme pour dire : «NON à des élections truquées et sans importance ». 

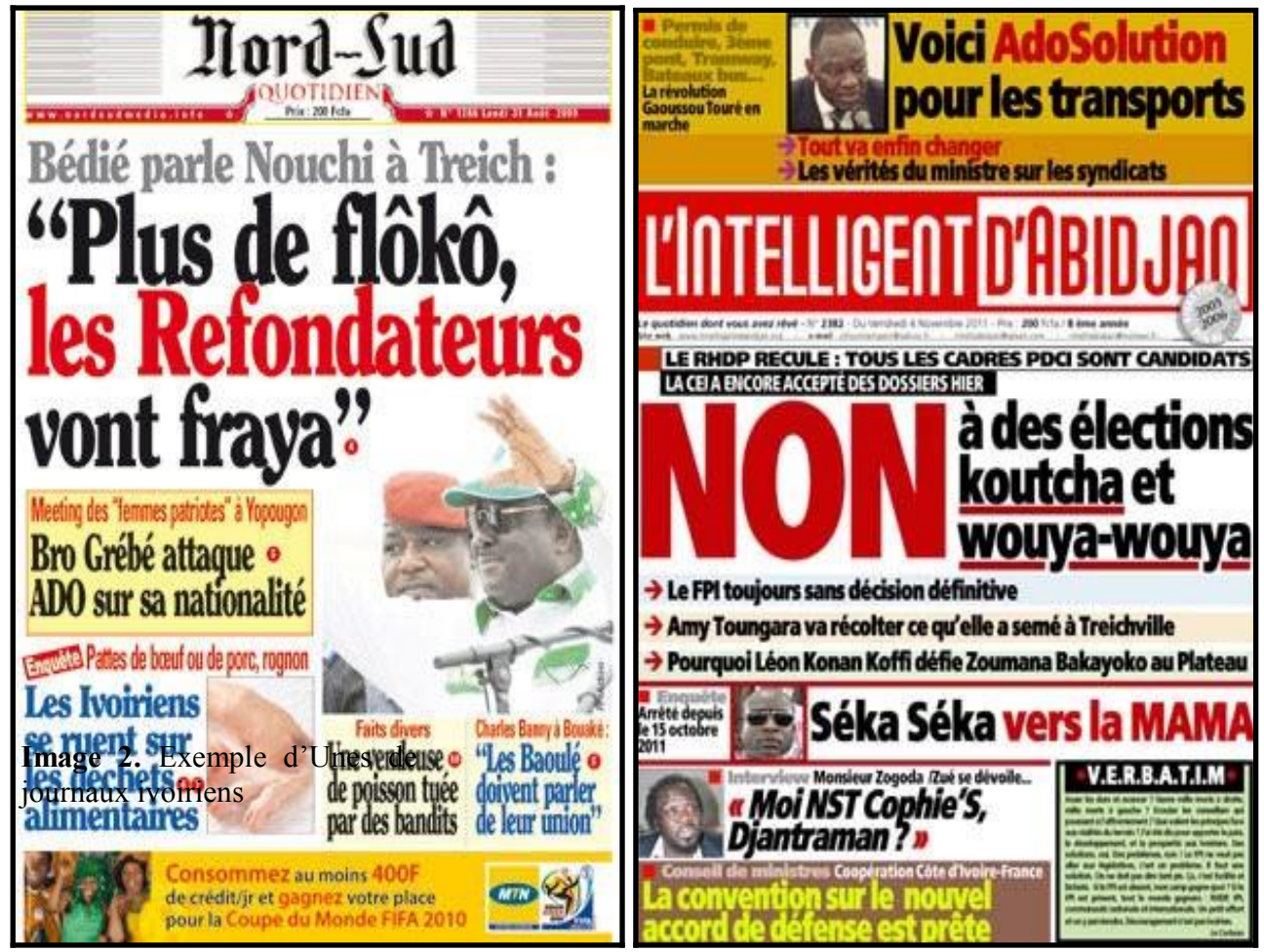

Image 2. Exemple d'Unes de journaux ivoiriens

Toujours dans le même cadre des élections, le nouchi est utilisé pour véhiculer un message à la population ivoirienne dominée majoritairement par des jeunes. Ainsi, pour l'élection présidentielle d'octobre 2015, une campagne publicitaire a invité certaines stars ivoiriennes du sport à sensibiliser la population en cette phrase : « élection c pas gnaga » juste pour dire qu'une « élection ce n'est pas de la bagarre » (Voir l'image 3 ci-après).

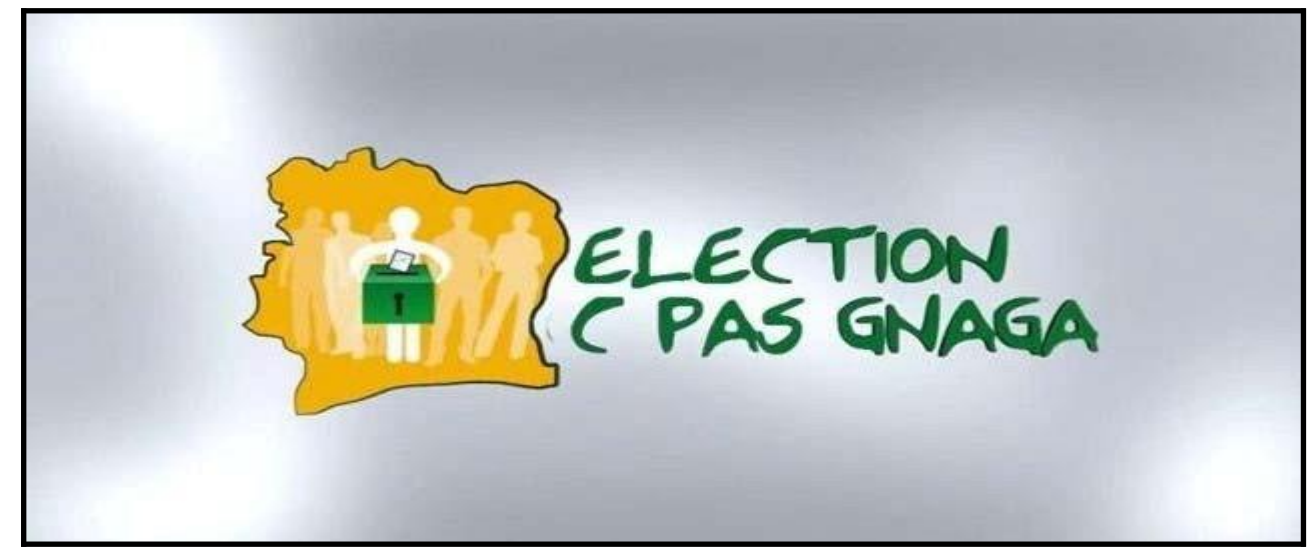

Image 3. Publicité servant de campagne pour les élections présidentielles de 2015 
Aujourd'hui, le nouchi occupe une place très importante sur les réseaux sociaux. Les internautes ivoiriens, entre un réel désir de fidélité à la culture ivoirienne et une non moins ardente envie d'épouser totalement et absolument cette nouvelle identité linguistique, donnent des cours en nouchi sur Facebook, Twitter, Instagram, etc. Ces deux images ci-dessous tirées des réseaux sociaux le démontrent.

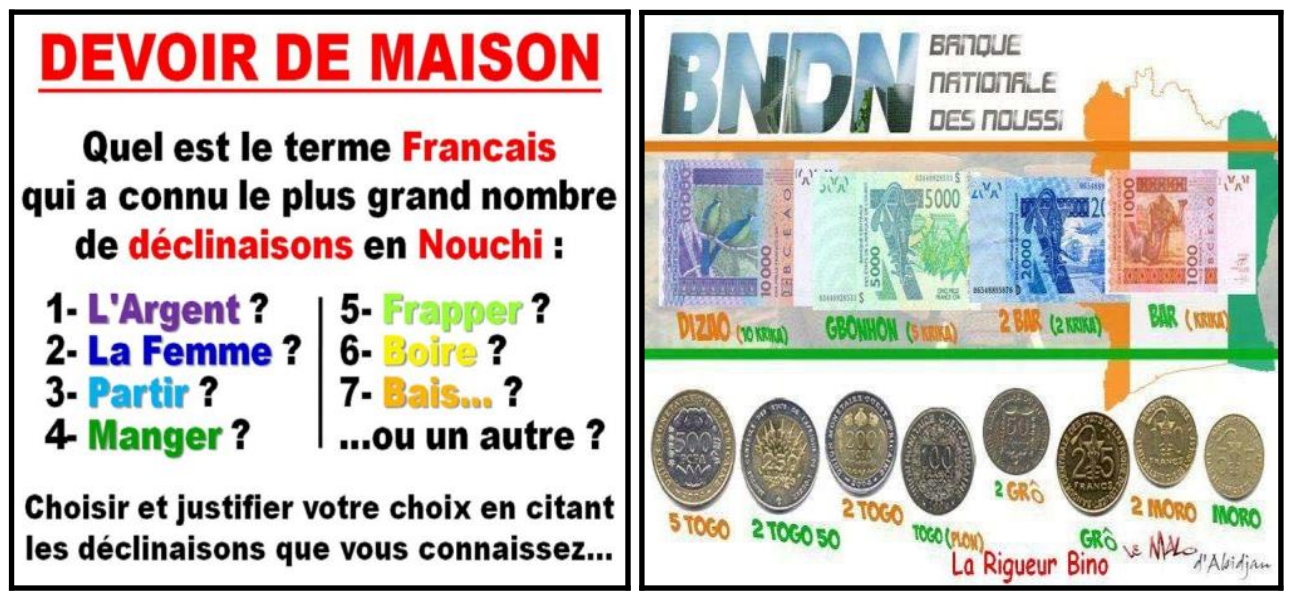

Images 4. Le nouchi sur les réseaux sociaux

La première image (Devoir de maison) recherche auprès des internautes des mots français qui ont plus de variantes en nouchi, tandis que la seconde (BNDN pour Banque Nationale Des Noussi) donne la signification en nouchi de [ses] quelques pièces et billets de banque.

Enfin, sur le plan culturel, précisément dans le domaine musical, grâce à leur imagerie populaire, leur humour et la façon simpliste d'écrire leurs textes et messages, de nombreuses tournures et expressions proverbiales nouchi comme taper dos (« cocufier »), montrer à quelqu'un qui a mis l'eau dans coco ("montrer à quelqu'un de quoi on est capable »), c'est naissance ( «C'est congénital ou c'est de nature génétique, en parlant de la forme physique de quelqu'un»), etc. ont été révélé au grand public par les chanteurs zouglou, comme c'est souvent le cas du groupe Magic System, Yodé et Siro, Petit Denis et bien d'autres. Dans les arts, l'utilisation du nouchi produit un plaisir esthétique lié à la satisfaction immédiate qu'éprouvent les récepteurs qui se reconnaissent dans cet univers. Les téléfilms et séries télévisées ivoiriens ne sont pas en reste. Depuis les années 1970-1980 dans les dialogues d'acteurs de sketches tels que Comment ça va?, puis ceux des séries télévisées Qui fait ça? et Faut pas fâché! dans les années 1990 et depuis 2000 avec Ma Famille, Safi, Dr Boris, Sicobois, Cour commune, etc. le nouchi a pris une proportion très importante au point de devenir la future langue nationale.

\section{Le nouchi, la future langue nationale de Côte d'Ivoire ?}

Un sociolecte qui occupe une partie de la nation a-t-il vocation à accéder au statut de langue nationale? À propos, Calvet annonce déjà ce que sera l'avenir du nouchi : 
De plus en plus fréquemment, en effet, lorsque les Ivoiriens se rencontrent à l'étranger, ils ont tendance à employer des mots ou des expressions venus du nouchi, comme pour marquer leur "ivoirité". Aujourd'hui forme identitaire la langue identitaire ivoirienne, le nouchi, nous donne donc à voir une tendance évolutive et préfigure sans doute ce que sera demain (je pense bien sûr à un lendemain historique) le français de Côte d'Ivoire. Une langue différente du français standard (Calvet, 1997, p. 33).

Sabine Kube, dans sa même enquête (2005, p. 142-146) menée auprès de quelques élèves d'Abidjan, recense trois critères évoqués par ceux-ci pour qu'une langue bénéficie du statut de langue nationale. Ce sont : le critère identitaire, le critère de la compétence linguistique et le critère de l'unification nationale.

Pour ces élèves, le nouchi répond au critère identitaire, car il exprime le mieux l'identité ivoirienne dans un pays multiethnique; il «symbolise leur identité vis-à-vis de l'extérieur et est une langue grâce à laquelle on reconnaît les Ivoiriens " (Kube, 2005, p. 145). En ce qui concerne le critère de la compétence linguistique, «une langue nationale doit permettre la communication interethnique, être une langue parlée et comprise dans tout le pays (...). Le français serait parlé par la plupart des Ivoiriens, mais n'est pas compris pas tout le monde », affirment les mêmes élèves (Kube, 2005, p. 144). Pour eux, c'est donc le nouchi qui est parlé et compris dans tout le pays et qui pourrait jouer ce rôle de communication interethnique. Aussi, le fait que les élèves cherchent, en quelque sorte, refuge dans cette variété linguistique qui ne respecte pas les règles du français standard est vu, selon Lafage (1996, p. 599), comme un résultat direct du recul de la compétence en français. Enfin, pour le critère de l'unification nationale, les élèves présentent le nouchi comme «une langue qui unit la population à l'intérieur du pays, une langue dans laquelle toute la population se reconnait; un critère qui n'est pas rempli par le français» (Kube, 2005, p. 145). L'on pourrait donc dire: "Le nouchi c'est notre créole en quelque sorte, qui est parlé par presque toute la Côte d'Ivoire » (Boutin et Kouadio, 2015).

Mais aujourd'hui, considérer le nouchi comme la langue nationale de la Côte d'Ivoire a aussi bien des avantages et des limites.

\subsection{Les avantages du nouchi comme langue nationale}

Dans certains débats scientifiques et intellectuels, les Ivoiriens montrent leurs aspirations pour une langue nationale comme c'est le cas chez certains de leurs voisins africains. Ils sont tous unanimes sur le fait que le nouchi est considéré comme l'un des symboles de l'identité ivoirienne [14]. Ce parler argotique a véritablement modifié la donne linguistique ivoirienne et répond bien aux critères tels que définis par les élèves abidjanais dans l'enquête de Kube (2005) et nos étudiants de l'université de Bouaké pour occuper la place de langue nationale, à savoir :

- Une langue typiquement et véritablement ivoirienne, une "invention" des Ivoiriens ;

- Une langue comprise et parlée par l'ensemble des Ivoiriens ;

- Une langue qui rassemble les Ivoiriens et qui est le mélange de plusieurs langues ivoiriennes.

L'un des avantages également du nouchi, c'est qu'il n'a jamais été imposé aux Ivoiriens, mais s'est imposé de lui-même au point qu'il est devenu une réalité sociale faisant désormais partie du patrimoine linguistico-culturel de la Côte d'Ivoire. Plusieurs recherches scientifiques (thèses de doctorat, mémoires, articles scientifiques et de presse, séminaires, conférences, etc.) ont été consacrées au nouchi dans toutes ses formes. En plus de son dynamisme, beaucoup reste à faire. 


\subsection{Les limites du nouchi}

Malgré quelques données à ses avantages, le nouchi a aussi des limites. L'un des inconvénients est son instabilité et la non uniformité de son code graphique. En effet, il est très difficile aujourd'hui pour les nouchiphones et les nouchiphiles d'écrire leurs messages avec les mots de ce parler. Ahua en essayant d'élaborer un code graphique pour le nouchi dans l'un de ses articles afin de permettre d'en assurer une meilleure communication écrite, se pose la question suivante :

«n'est-il pas malgré tout précoce d'élaborer un code graphique d'une jeune langue en pleine expansion, et dont on n'a pas encore cerné tous les contours? » (Ahua, 2007, p. 183). Sa question annonce déjà les difficultés que rencontrent les adeptes de cet argot à l'écrire aisément pour le besoin de la communication, car tous les contours ne sont pas encore cernés. On découvre une véritable instabilité structurelle (morphosyntaxique) et surtout formelle (lexicale). Comme exemple, un mot en nouchi pour dire "parler; discourir publiquement» pourrait s'écrire gbahé ou gbayé. Certains, en plus de l'écrire avec un $" \mathrm{~h} »$ ou un $" \mathrm{y} »$, écriraient la terminaison avec «ER» (gbaher ou gbayer) par analogie aux infinitifs des verbes français du premier groupe. Il en est de même du mot $d r a$ dans l'expression ya dra (il y a des problèmes) où les nouchiphiles l'écriraient avec ou sans le [p] en faisant référence au mot drap français.

Au niveau syntaxique, l'une des limites du nouchi, c'est de jouer sur le déterminant du mot. Là où la langue française emploierait l'article défini «la » pour le genre féminin, le nouchi utilise plutôt le genre masculin, comme dans les exemples suivants :

Le gomi / la gomi pour

«jeune fille »

Le piair ; le pia (la pierre)

pour «l'argent»

L'un des problèmes du nouchi renvoie à son vocabulaire hétéroclite et composite. Ses mots sont d'origines diverses avec des emprunts aux langues européennes (le français, l'anglais et l'espagnol en particulier), ivoiriennes (le dioula, le baoulé, le bété, l'attié, etc.), avec des mots créés par un processus onomatopéique et idéophonique et également des mots le plus souvent issus du ghetto et d'origine inconnue. De tels procédés de création de mots utilisés par les nouchiphones font du nouchi une langue spécifique avec un code graphique multiforme, quand nous savons que la plupart des langues ivoiriennes ne sont pas écrites. En réalité, le nouchi est plus oral et gestuel que graphique. C'est pourquoi la graphie d'un mot diffère d'un scripteur à un autre. Exemple [15] :

(1)- $<$ quechia $>,<$ késhia $>,<$ kèssiah $>,<$ kessia $>$ pour «

Qu'est-ce qu'il y a » (2)- < $<$ môgô $>,<\operatorname{mog} \hat{\text { }}>,<\operatorname{mogo}>$, signifiant "individu, ami"

(3)- <béou $>,<$ béhou $>,<$ behou $>$, signifiant

"partir, quitter" (4)- < pierre>, < piair $>$,

signifiant "argent"

D'autre part, ce qui serait également désavantageux pour le nouchi en tant que langue nationale, c'est le fait qu'il découle du français en tant que variété. Or le français étant la langue officielle de la Côte d'Ivoire et de certains pays d'Afrique et d'Europe, il ne serait pas du tout convenable pour les Ivoiriens de partager avec d'autres pays une langue qui serait «leur langue nationale». Kube en témoigne : 
Ils citent d'autres pays africains comme le Mali ou le Sénégal où une langue africaine s'est développée comme langue nationale à côté d'une langue officielle européenne. Les Maliens seraient reconnus partout grâce au bambara et les Sénégalais grâce au wolof. Un étranger qui viendrait dans ces pays apprendrait ces langues pour pouvoir communiquer avec les habitants. Cet effort ne serait pas nécessaire dans le cas de la Côte d'Ivoire parce que tout le monde partirait du principe qu'une intercompréhension est possible grâce au français. (...) D'autres propos d'élèves insistent aussi sur le fait qu'une langue nationale devrait en quelque sorte différencier les nationaux des étrangers (Kube, 2005, p. 145).

Enfin, les mots et expressions qui composent le nouchi sont créés pêle-mêle et de façon non univoque. Et on finit par s'en rendre bien compte que «le ou les parlers nouchi sont en effet renouvelé(s) en permanence, délaissant les termes et les expressions devenus trop populaires...» (Mel et Kouadio, 1990, p. 53). Des mots comme gaou ( «stupide ; niais »), on dit quoi ? («Comment vas-tu? ; Quoi de neuf? »), choco (« branché ; à la mode ») ou s'enjailler [16] sont des classiques. D'autres comme sao, toutou ("pute », "prostituée »), par contre, sont aujourd'hui moins en vogue au profit de leurs synonymes djandjou, djantra, pinhou, kpôklé. Mais, loin d'être des limites, tous ces exemples pourraient confirmer la capacité du nouchi à évoluer tant à diachronie qu'en synchronie dans leur contexte de production pour le bonheur des Ivoiriens.

\section{Conclusion}

L'appropriation du français en Côte d'Ivoire a donné ainsi naissance à différentes variétés dont le nouchi, la variété la plus usitée. Autrefois réservé à des jeunes délinquants des milieux défavorisés d'Abidjan, le nouchi ne s'identifie plus à un groupe, car il a acquis ses lettres de noblesse. Son utilisation dans toutes les sphères de la vie publique et privée en Côte d'Ivoire est aujourd'hui considérée comme une sorte de légitimation de cette forme d'expression. Aujourd'hui, ce sociolecte a fini par convaincre par son rôle véhiculaire indéniable, en s'imposant, non seulement dans le paysage socio-linguistique de la Côte d'Ivoire, mais également en tant que vecteur des valeurs culturelles et en tant que canal d'expression, de rassemblement et de revendications identitaires des Ivoiriens. Pour certains chercheurs, "ses différents usages, parce qu'ils créent de nouvelles valeurs sur le plan sémantique, favorisent un enrichissement qui pourrait conduire à une langue à part entière ». Mais également, nous estimons que les mass-médias (radios, télévisions, presses écrites), la politique, la culture (chansons, téléfilms, littérature) et même la publicité « contribuent, par la même occasion, à faire du nouchi une langue d'intégration, un ciment social, l'outil d'une identité qui se construit à l'échelle de la nation [17]».

Enfin, dans un contexte africain plurilingue comme la Côte d'Ivoire caractérisé par l'absence d'un véhiculaire d'envergure nationale, le nouchi ne vient-il pas combler un manque ? Ne correspond-il pas à un besoin pour les Ivoiriens d'avoir une langue qu'ils comprennent et parlent tous aux quatre coins du pays ? Si tel est le cas, le nouchi pourrait devenir la " langue nationale » de la Côte d'Ivoire du fait de ses attributs identitaires, de compétence linguistique et d'unification nationale, mais beaucoup reste à faire : harmonisation de la graphie des mots et du lexique et des différentes parties du discours. Dans les perspectives, il serait donc intéressant de mener une autre réflexion sur ces aspects et d'en faire l'objet d'un travail de recherche. 


\section{Références}

1. Aboa, A. A. Le nouchi a-t-il un avenir? Sudlangues 16, Dakar, p. 44-54, (2011).

2. Ahua, M. B. Élaborer un code graphique pour le nouchi : une initiative précoce ? Le Français en Afrique 22, p. 183-198 (2007).

3. J.-B. Atsé N'Cho, Les verbes du nouchi (parler argotique ivoirien) : pour une analyse morphosyntaxique. Revue du Laboratoire des Théories et Modèles Linguistiques (LTLM) 10, Institut de Linguistique Appliquée, Université Félix Houphouët-Boigny,Abidjan,(2014).

En ligne, Url: http://www.ltml.ci/files/articles10/Jean-BaptisteATSeNCHO.pdf

4. J.-B. Atsé N'Cho, Francophonie ivoirienne et créativité lexicale : comment "fabrique-t-on" les mots en nouchi ? Rachele RAUS \& Laurentiu BALA (coordination) Sur l'argot au XXIè siècle, Editura Universitaria Craiova, Roumanie, p. 93-109 (2016).

5. D. H. Bohui, Petit recueil d'ivoirismes. Paris, Publibook, 121p, (2013).

6. A. B. Boutin, Description de la variation: Etudes transformationnelles des phrases du français de Côte d'Ivoire. Thèse de doctorat. Université de Grenoble 3. Villeneuve d'Ascq, Presses Universitaires du Septentrion, (2002).

7. A. B. Boutin, J. Kouadio N'guessan, Le nouchi c'est notre créole en quelque sorte, qui est parlé par presque toute la Côte d'Ivoire. Blumenthal, P. (éd.), Dynamique des français africains : entre le culturel et le linguistique. Berne, Peter Lang, p. 251-271, (2015).

8. L-J. Calvet, La sociolinguistique, Paris, PUF, Coll. Que sais-je ?, (1993).

9. L-J. Calvet, Le nouchi, langue identitaire ivoirienne? Diagonales 42, p. 33, (1997).

10. R. Chaudenson, Mondialisation : la langue française a-t-elle encore un avenir? Paris, L'Harmattan. (2000).

11. J.-C. Dodo, Le nouchi : étude linguistique et sociolinguistique d'un parler urbain dynamique. Thèse de doctorat unique, Département des Sciences du langage, Université Félix Houphouët-Boigny, Abidjan, Côte d'Ivoire, (2015).

12. P. Dumont, \& B. Maurer, Sociolinguistique du français en Afrique francophone. Gestion d'un héritage, devenir d'une science, Paris, EDICEF, AUPELFUREF, (1995).

13.F. Gadet, La variation sociale en français, Nouvelle édition revue et augmentée. Paris : Ophrys, (2003).

14. N. J. Kouadio, Le nouchi abidjanais, naissance d'un argot ou mode linguistique passagère. Gouaini/Thiam (éds.), Des Langues et des villes. Actes du colloque international «Des langues et des villes », Paris, décembre 1990. Paris, Didier Erudition, p. 373-385, (1991).

15. N. J. Kouadio, Quelques traits morphosyntaxiques du français écrit en Côted'Ivoire. Cahiers d'études et de recherches francophones, Langues. II, 4, p. 301314, (1999).

16. N. J. Kouadio, Le français et la question de l'identité culturelle ivoirienne. Pierre Dumont et Christine Santodomingo (éds. 2000), La coexistence des langues dans l'espace francophone, approche macrosociolinguistique. Paris, AUF, p. 199207, (2000).

17. N. J. Kouadio, Le nouchi et les rapports dioula-français. Des inventaires lexicaux du français en Afrique à la sociologie urbaine... Hommage à Suzanne Lafage. Le français en Afrique, 21. Nice, ILF - CNRS, p. 177-191, (2006). 
18. K. J.- M. Kouamé, Vers une généralisation du parler jeune de Côte d'Ivoire. $L a$ revue des Lyriades de la Langue française, 1, Angers, p. 70-76, (2013).

19. S. Kube, La francophonie vécue en Côte d'Ivoire, Paris, L'Harmattan, 247 p., (2005).

20. S. Lafage, Description sommaire de la situation sociolinguistique de la Côte d'Ivoire. CONFEMEN Promotion et intégration des langues nationales dans les systèmes éducatifs. Bilan et inventaire, 90, Paris, Champion, p. 501-517, (1986).

21. S. Lafage, L'argot des jeunes Ivoiriens, marque d'appropriation du français ? Langue française, 90, Larousse, Paris, p. 95-105, (1991).

22. S. Lafage, La Côte d'Ivoire : une appropriation nationale du français ? Robillard, Didier de et Michel Beniamino (éds.) Le français dans l'espace francophone. 2 , p. 597-602, (199).

23. S. Lafage, Hybridation et "français des rues" à Abidjan. Alternances codiques et français parlé en Afrique, Publications de l'Université de Provence, Aix-enProvence, p. 279-291, (1998).

24. S. Lafage, Le lexique français de Côte-d'Ivoire. Appropriation \& créativité, Le Français en Afrique Noire, Revue du ROFCAN, 16 et 17, tomes 1 et 2, (2002-2003).

25. G. B. Mel, N. J. Kouadio, Variétés lexicales du français en Côte d'Ivoire. Sous la direction d'André CLAS et Benoît OUOBA Visages du français. Variétés lexicales de l'espace francophone, Edition Jogn Libbey Eurotext - AUPELF, Coll.

Actualités scientifiques, p. 51-58, (1990).

26. G.Manessy, Le français en Afrique noire. Mythes, stratégies, pratiques, L'Harmattan, Paris, 245 p., (1994).

27. M.-L. Moreau, N. Thiam et C. Bauvois, Le marquage identitaire dans le français d'Afrique. Etude exploratoire au Sénégal. Louis-Jean Calvet et Marie-Louise Moreau (éds. 1998): Une ou des normes? Insécurité linguistique et normes endogènes en Afrique francophone, Paris, Didier Erudition, p. 111-127, (1998).

28. A.Queffelec, Les parlers mixtes en Afrique francophone subsaharienne. Le Français en Afrique 22, p. 277-291, (2007).

29. A.Sangaré, Peuples et langues de l'Afrique de l'ouest comme éléments dynamiques d'une intégration réussie. Yéo S. S. (eds), Les Etats-nations face à l'intégration régionale en Afrique de l'ouest. Le cas de la Côte d'Ivoire, Karthala, Paris, p. 207-224, (2009).

30. S .Soro, (Coulibaly, S., alias). Zouglou et nouchi, les deux fleurons pervertis de la culture urbaine. Africultures, 56, p. 123-124, (2003).

31. P.Wald, L'appropriation $\mathrm{du}$ français en Afrique noire : une dynamique discursive. Langue française, 104, p. 115-124, (1994).

32. H.Walter, Le français dans tous les sens, Paris, Robert Laffont. (1988).

\section{Notes}

[1] Le Zouglou est un genre musical urbain et populaire né dans les cités universitaires d'Abidjan dès l'avènement du multipartisme en Côte d'Ivoire dans les années 1990.

[2] Joseph H. Greenberg (1963). The Languages of Africa, International Journal of American linguistics.

[3] Chaudenson fait une différence terminologique entre le "plurilinguisme » et le " multilinguisme ». Pour lu i, la diversité linguistique au sein d'un État serait ainsi appelée "plurilinguisme", tandis que la présence des mêmes langues dans plusieurs serait appelée "multilinguisme" (Chaudenson, 2000, p. 25-26). 
[4] - la famille GUR avec 7 langues : le birifor parlé en Côte d'Ivoire, Burkina Faso et au Ghana ; le degha (Côte d'Ivoire, Ghana) ; le lobiri (Côte d'Ivoire, Burkina Faso) ; le lohron (Côte d'Ivoire, Ghana); le koulango (Côte d'Ivoire, Ghana); le senoufo (Côte d'Ivoire, Burkina Faso, Ghana, Mali) et le gouin (Côte d'Ivoire, Burkina Faso). - la famille KROU avec 3 langues : le grébo (Côte d'Ivoire, Libéria); le kroumen (Côte d'Ivoire, Libéria) et le wè (Côte d'Ivoire, Libéria). - la famille KWA avec également 3 langues : l'abron (Côte d'Ivoire, Ghana) ; l'agni-baoulé (Côte d'Ivoire, Ghana, Togo) et le n'zima (Côte d'Ivoire, Ghana). - la famille MANDÉ, aussi avec 3 langues : le dan aussi appelé yacouba est parlé en Côte d'Ivoire et au Libéria ; le ligbi (Côte d'Ivoire, Ghana) et le mandingue (Côte d'Ivoire, Burkina Faso, Ghana, Guinée, Mali).

[5] Le manding représente en Côte d'Ivoire les langues malinké, bambara et dioula, mais pris ici sous la forme du dioula.

[6] Ici, pour des cas de similitudes, certains chercheurs comme Lafage (1986), Sangaré (2009), etc. préfèrent réunir l'agni et le baoulé en une seule langue.

[7] Cf. KOUADIO N'guessan Jérémie, dans sa Préface à l'ouvrage Petit recueil d'ivoirismes de Bohui Djédjé Hilaire (2013, p. 9-10).

[8] Les nouchiphones ne font pas l'amalgame entre le nouchi qui représente la langue (le parler) ; le ou la Noussi celui ou celle qui parle le nouchi, qui l'utilise au quotidien (c'est-àdire le locuteur) et le noussiya ou le nouchiya qui est admis dans leur entendement comme étant le style et tous les aspects comme la prononciation et la gestuelle qui vont avec ce parler argotique (Cf. Atsé N'Cho, $2014 ; 2016)$.

[9] Cf. Kouadio N'guessan Jérémie, dans sa Préface à l'ouvrage Petit recueil d'ivoirismes de Bohui Djédjé Hilaire (op. cit. p. 11).

[10] Dans leur publication sur la politique linguistique en Afrique, Heine, Bernd et Reh, Mechthild (1982, cité par Sabine Kube), affirment que lorsque la langue officielle est une langue européenne, la politique linguistique est appelée exoglossique (Kube, 2005 : 75).

[11] Cf. Rapport de la Commission 2 de l'Atelier sur le thème « Le nouchi et les arts de la parole : quelles interactions? » dans les Actes du séminaire sur «Le nouchi, mal de vivre de la jeunesse ou alternative possible d'une identité ivoirienne en construction? » tenu du 17 au 19 juin 2009 à Grand-Bassam (Côte d'Ivoire), p. 37.

[12] Nous empruntons les transcriptions de ce texte à Kouadio (2000, p. 202-203), mais que nous avons légèrement modifiée pour le besoin de l'étude.

[13] Pour Kouadio (2000, op. cit., citant l'IFA, p. 79), Faire « chocobit » (ou « chocobi »), c'est essayer de prononcer comme un Français alors qu'on est Africain.

[14] Sous l'égide du ministère ivoirien de la Culture et de la Francophonie, un séminaire réunissant plusieurs scientifiques, intellectuels et professionnels du secteur des arts et de la culture a été organisé du 17 au 19 juin 2009 à Grand-Bassam (Côte d'Ivoire), sur le thème : " Le nouchi, mal de vivre de la jeunesse ou alternative possible d'une identité ivoirienne en construction?».

[15] Ces exemples sont tirés de AHUA Mouchi Blaise (2007, p. 184)

[16] Mot tiré du verbe anglais "to enjoy" (apprécier) qui signifie en nouchi "s'amuser" ou "se faire plaisir". Il fait partie des 150 nouveaux mots, sens, locutions et expressions qui ont fait leur entrée dans le dictionnaire Le Petit Larousse 2017 mis en vente depuis le 26 mai 2016.

[17] Voir les conclusions de la Commission 2 dans le rapport général du séminaire sur le nouchi du 17 au 19 juin 2009 à Grand-Bassam (Côte d'Ivoire) op. cit. p. 57. 